\title{
Full-energy, vacuum-compatible, single-shot pulse characterization method for petawatt-level ultra-broad bandwidth lasers using spatial sampling
}

\author{
B. Webb, S. W. Bahk, I. A. Begishev, C. Dorrer, C. Feng, C. Jeon, M. Spilatro, R. Roides, J. Zuegel, \\ and J. Bromage \\ Laboratory for Laser Energetics, University of Rochester, 250 East River Road, Rochester, NY, 14623-1299, USA
}

Optical parametric chirped-pulse amplification (OPCPA) enables the amplification of ultra-broad bandwidths beyond the petawatt level, creating the potential for shorter pulse durations than are achievable with traditional CPA. Since these shorter pulses are highly sensitive to material dispersion, all-reflective optics are typically used after the pulse compressor to ensure that the pulse characterized by a diagnostic is the same as the pulse interacting with the target. Because beam splitters, leaky mirrors, and other sampling optics introduce chromatic dispersion and nonlinear distortion, sampling the beam to measure the pulse duration on target at full energy is problematic.

In this work we investigate the accuracy of spatially sampling the beam in order to provide single-shot pulse duration on target at full energy with spectral phase interferometry for direct electric-field reconstruction (SPIDER) [1]. This technique was utilized in the first-light experiments of a new 0.5-PW, 20-fs OPCPA system that was completed in 2020, the Multi-Terawatt-pumped Optical Parametric Amplifier Line (MTW-OPAL) [2]. Precise apodization of a beamlet near the edge of the main $90-\mathrm{mm}$ beam before the compressor using a spatial dithering, allows for a small mirror after the compressor to pick off the beamlet for pulse characterization with only $2.5 \%$ loss in overall energy to target (Fig. 1). This method of spatial sampling allows for pulse-shape measurement at the same time the full-energy beam is interacting with the target, providing on-shot pulse shape information for experiments. Vacuum compatibility of a single-shot SPIDER is accomplished by placing spectrometers for the fundamental and upconverted SPIDER signal outside the vacuum chamber. Transmission through a window is permitted after the nonlinear crystal since the spectral phase has already been encoded in the up-converted interferogram.

The local pulse duration is measured at several points across the beam to verify the accuracy of this method. Since this spatially resolved characterization detects changes in dispersion and not group delay, the far-field spot was also characterized to detect the presence of spatiotemporal distortions [3]. Furthermore, the full-aperture beam is characterized at low energy with another SPIDER device and compared to sub-aperture measurement to determine the accuracy of this spatial sampling method. Periodic full-aperture measurements combined with a new spatiotemporal diagnostic [4] can maintain the accuracy of this spatial sampling method over time.

This material is based upon work supported by the Department of Energy National Nuclear Security Administration under Award Number DE-NA0003856, the University of Rochester, and the New York State Energy Research and Development Authority. The support of DOE does not constitute an endorsement by DOE of the views expressed in this abstract.

(a)

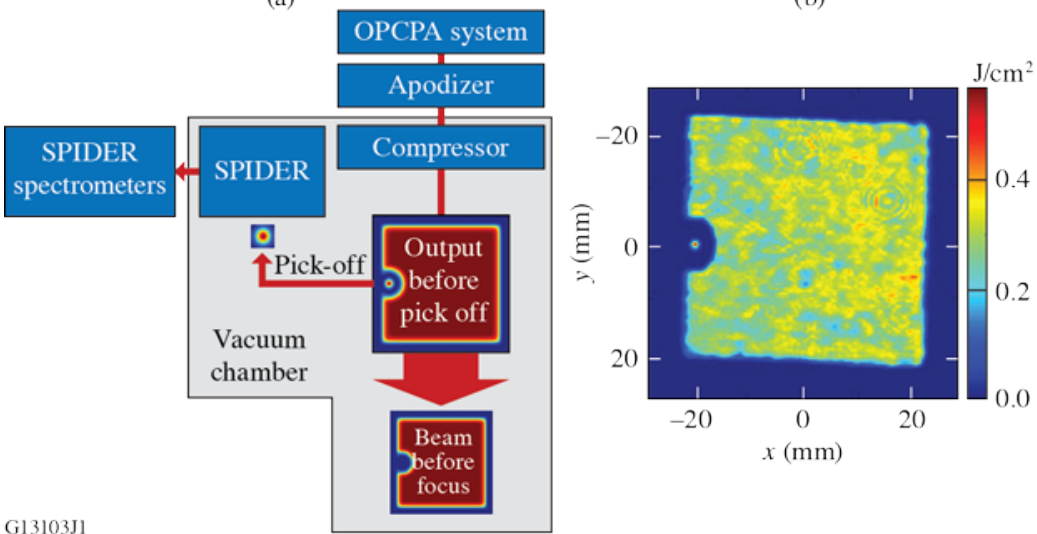

(c)

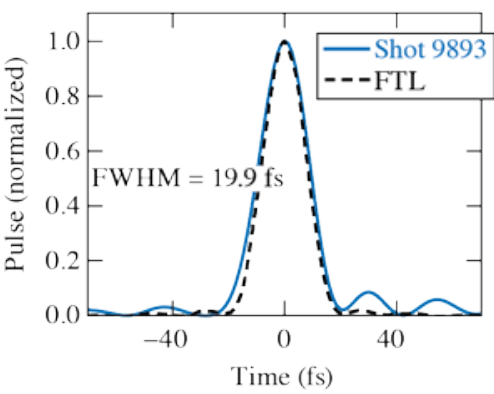

Fig. 1. (a) Schematic of the MTW-OPAL system showing the back end. (b) Output apodized beam profile before the pulse compressor. (c) Pulse calculated from SPIDER measurement compared to the Fourier-transform limit (FTL).

\section{References}

[1] C. Dorrer, B. de Beauvoir, C. Le Blanc, S. Ranc, J.-P. Rousseau, P. Rousseau, and J.-P. Chambaret, "Single-Shot Real-Time Characterization of Chirped-Pulse Amplification Systems by Spectral Phase Interferometry for Direct Electric-Field Reconstruction,” Opt. Lett. 24, 1644 (1999).

[2] J. Bromage, S.-W. Bahk, I. A. Begishev, C. Dorrer, M. J. Guardalben, B. N. Hoffman, J. B. Oliver, R. G. Roides, E. M. Schiesser, M. J. Shoup III, M. Spilatro, B. Webb, D. Weiner, and J. D. Zuegel, “Technology Development for Ultraintense All-OPCPA Systems,” High Power Laser Sci. Eng. 7, e4 (2019).

[3] C. Dorrer, “Spatiotemporal Metrology of Broadband Optical Pulses,” IEEE J. Sel. Top. Quantum Electron. 25, 3100216 (2019).

[4] S.-W. Bahk, C. Dorrer, R. G. Roides, and J. Bromage, “Chromatic-Aberration Diagnostic Based on a Spectrally Resolved Lateral-

Shearing Interferometer,” Appl. Opt. 55, 2413 (2016). 